\title{
Militares y conservación de la naturaleza en el Perú
}

\author{
Military and nature conservation in Peru
}

\author{
Marc J. Dourojeanni $\square$ \\ Universidad Nacional Agraria de La Molina. Lima, Perú
}

Recibido: 22/10/2020

Revisado: $15 / 11 / 2020$

Aceptado: 20/11/2020

Publicado: 16/12/2020

\section{RESUMEN}

Es común no considerar que gobiernos militares también adoptaron medidas importantes para la conservación del patrimonio natural. Sin embargo, la realidad en el Perú y en el Brasil indica que parte significativa de las áreas naturales protegidas, así como leyes y otras medidas para proteger la biodiversidad, fueron realizadas durante esas interrupciones de la democracia. En esta nota se destaca la actuación del general Enrique Gallegos Venero en el Perú. Palabras clave: Gobiernos militares, historia, conservación de la naturaleza, áreas protegidas.

\begin{abstract}
It is common not to consider that military governments also adopted important measures for the conservation of natural heritage. However, the reality in Peru and Brazil indicates that a significant part of the protected natural areas, as well as laws and other measures to protect biodiversity, were carried out during these democracy interruptions. This note highlights the performance of General Enrique Gallegos in Peru.
\end{abstract} Keywords: Military governments, history, nature conservancy, protected areas.

\section{INTRODUCCIÓN}

Gobernar no es función de los militares. Cuando ellos asumen el gobierno es porque han usurpado el poder. Y, por eso, se asume que sus acciones como gobernantes son dictatoriales o tiránicas y por ende inválidas o despreciables. Por eso, muchas veces los que escriben la historia no llevan en cuenta o, más bien, prefieren olvidar hechos importantes para el futuro de la nación que se producen en esos lapsos. Por ejemplo, no suele recordarse que militares peruanos, en diversos gobiernos tomaron medidas de gran importancia para la conservación del patrimonio natural del Perú. En esta nota el autor va a destacar la actuación del general Enrique Gallegos Venero, a quien se debe en última instancia el establecimiento de parques, reservas y santuarios nacionales como Huascarán, Cerros de Amotape, Huayllay, Paracas y Junín, entre otras así como el auge de los programas de conservación de la vicuña y de primates, la inauguración de un curso de "ecología y defesa nacional" $y$, entre otras obras, la promulgación de la Ley Forestal y de Fauna 21147, que fue la primera que trató extensa y detalladamente de las áreas naturales protegidas. 


\section{El proceso de las decisiones del gobierno}

Es evidente que las grandes decisiones nacionales sobre conservación de la naturaleza, como otras de interés público, son finalmente adoptadas por los gobiernos de turno $\mathrm{y}$, por ende, atribuidas a las autoridades que, mediante sus firmas en leyes, ordenan sean cumplidas. Pero, como bien se sabe, la génesis de esas decisiones frecuentemente no guarda relación con el gobierno que finalmente las transforma en dispositivos legales. Puede corresponder a la gestión anterior $\mathrm{o}$, también, es frecuente que los gobiernos y autoridades o sectores que las aprueban sean los mismos que hicieron todo lo posible para evitarlas o limitarlas y que, terminen adoptándolas apenas respondiendo a las presiones o, simplemente, porque en verdad, no les importa el tema. Esta es la realidad que precede gran parte de las más importantes decisiones nacionales sobre el ambiente, como en el caso del establecimiento de áreas naturales protegidas. Sin embargo, existen casos en que esas iniciativas son del propio gobierno, como ocurrió con más frecuencia a partir de la creación del Ministerio del Ambiente o en los que las iniciativas son realmente adoptadas oficialmente. Por eso, antes de atribuir las realizaciones ambientales como mérito de los gobiernos que a lo largo de la historia las materializaron, es preciso analizar cada caso.

Ese proceso, en el caso de las áreas naturales protegidas, es comúnmente muy largo y requiere de muchos actores, entre ellos algunos indispensables. Estos son de tres categorías: ideadores, proponentes y promotores. Es obvio que si no existe una idea nada comienza. Esa idea puede ser de uno o de pocos, muchas veces científicos soñadores o aficionados defensores de la naturaleza. Para que la idea comienza a andar debe ser procesada en forma de propuesta que después da lugar a un proyecto concreto. Sin ese instrumento, aunque sea embrionario, la idea no entra al proceso burocrático que permitirá materializarla. Los ideadores suelen no ser los que hacen la propuesta inicial ni, tampoco, se convierten en promotores, aunque hay casos raros en que tales roles se junten en uno o muy pocos personajes. Los promotores son los que tienen la tarea más dura, larga y compleja. En general ellos son miembros de nivel medio de las burocracias que deben desarrollar y empujar la propuesta. A ellos corresponde enfrentar las oposiciones, refutar las críticas, explicar los beneficios, convencer a los incrédulos, buscar apoyo, mantener el interés latente, ajustar una y otra vez la propuesta, negociar cada elemento de la misma y a veces ceder, aprovechar las oportunidades. Y, por encima de todo, deben tener fe en lo que desean hacer y mucha perseverancia.

Hay otros actores involucrados, unos a favor como puede serlo la prensa y otros en contra. En periodos democráticos los actores que están contra la propuesta, en el propio gobierno, donde aparecen muy fuertes los intereses sectoriales, o en la sociedad, pueden llevar el debate hasta el Congreso y en las denominadas consultas públicas. En esas circunstancias cualquier proyecto es tironeado por todos los lados a causa de la ignorancia y, en especial, para satisfacer los intereses de grupo o personas. En periodos militares eso también ocurre, debiendo la iniciativa pasar por múltiples consejos, comités y comisiones que filtran el proyecto antes de llegar a la firma. Pero en esos casos la discusión es más comedida. Hay cierto respeto por la autoridad y, a falta de argumentos serios, es mejor quedarse callado.

Sin embargo, todo el esfuerzo realizado por ideadores, proponentes y promotores, por heroico y constante que sea, puede fracasar si no encuentra un cuarto tipo de actor, también indispensable. Se hace referencia al padrino 
que adopta el proyecto. Este es necesariamente una personalidad situada en un lugar muy alto de la jerarquía gubernamental o política y con influencia. Esa personalidad es la que brinda el empujón final al proyecto. Ese fue el papel del general Enrique Gallegos Venero, cuándo fue ministro de agricultura durante el gobierno militar de Juan Velasco Alvarado, entre el seis de noviembre de 1974 y el 15 de julio de 1976, que permitió que tantas acciones de gran importancia ambiental se realizaran.

\section{Enrique Gallegos Venero: El general civilizado} Hasta hoy no sé gran cosa de Enrique Gallegos Venero (figural). Era cusqueño, pero desde muy joven había residido en Lima donde en 1946 se formó como oficial de infantería del ejército. Asimismo, por haberlo relatado él mismo, sé que había recibido entrenamiento militar en Francia y, por eso, también practicó en Argelia. Me consta que hablaba muy bien el francés además del inglés.

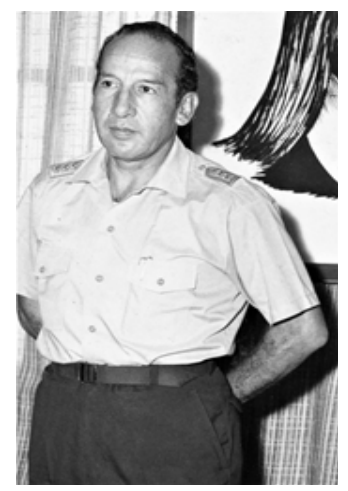

Figura 1. El general Enrique Gallegos Venero, ministro de agricultura en 1974.

Hasta hoy no sé gran cosa de Enrique Gallegos Venero. Era cusqueño, pero desde muy joven había residido en Lima donde en 1946 se formó como oficial de infantería del ejército. Asimismo, por haberlo relatado él mismo, sé que había recibido entrenamiento militar en
Francia y, por eso, también practicó en Argelia. Me consta que hablaba muy bien el francés además del inglés. Se sabe que en 1963 se encargó a Gallegos, siendo teniente coronel, la gerencia del nuevo Instituto de Reforma Agraria y Colonización (Irac) en La Convención, donde también debió asumir el control de la revuelta campesina en la que actuaba Hugo Blanco, en los valles de La Convención y Lares en la época del primer gobierno de Fernando Belaúnde Terry (Toche, 2008). Esa experiencia, según sus propias declaraciones, lo había impresionado y sensibilizado mucho en relación con la realidad campesina. Gustaba de la historia militar y, cuándo podía, describía maravillosamente las grandes hazañas militares. Además, escribiendo esta nota descubri que es autor de algunos libros sobre esos temas ${ }^{1}$. Y, también, parece haber sido un ideólogo dentro de las fuerzas armadas con varios artículos sobre lucha contra la subversión. Además, era un buen pintor, creo que especialmente de paisajes con acuarelas. Como todos, sé que él llegó a la fama... o a la infamia, porque fue uno de los coroneles cercanos al general Juan Velasco Alvarado que el 3 de octubre de 1968 condujo sus tanques livianos contra las rejas de Palacio de Gobierno para sacar de ese lugar al elegante arquitecto presidente.

Mas, en verdad, el 6 de noviembre de 1974, no sabía nada de Gallegos. Ese fue el día en que se convirtió en mi jefe directo. Es decir, fue designado ministro de agricultura. No fue el primer general en ese ministerio. Apenas desde el comienzo del golpe de Velasco ya habían pasado por ese cargo tres generales. Pero este ministro fue diferente a los que le precedieron y sucedieron. En efecto. Gallegos, como

${ }^{1}$ Un Siglo de Luchas, 1863-1962 (1999), El militar y el Retirado (1999), Cumbres y Abismos de la Vida (1998). 
se puede deducir por lo que ya dije sobre él, era definitivamente un general distinto. Yo lo llamaba, en mi círculo personal, de "general civilizado". Era realmente un hombre culto. Además, era sonriente, modesto y respetuoso. De esos que nunca permitía que un visitante esperara más de diez minutos a la entrada de su despacho y que, si realmente no podía cumplir, salía personalmente a pedir disculpas.

Gallegos no había sido ministro antes. Quizá por ser ese su primer cargo político importante o porque siempre fue así, lo tomó muy en serio, con entusiasmo juvenil, con muchas ganas de "hacer" y, por cierto, un tanto ingenuamente. Él llegó en un momento muy tumultuoso de la historia del sector agrario, sacudido durante los meses previos por el escándalo desatado en torno a la Empresa Pública de Servicios Agropecuarios (Epsa) ${ }^{2}$ que llevó a la cárcel a decenas de profesionales honestos como consecuencia de la ineptitud de algunos, que confundieron y malinterpretaron los hechos, y de la cólera irreflexiva de un dictador todopoderoso que, lleno de ira, ordenó "metan presos a todos" ... y así fue. El ministro anterior, el general Enrique Valdez Angulo, había sido en cierta forma responsabilizado por el presidente y, por eso, fue públicamente maltratado, pero, curiosamente, permaneció por varias semanas en el cargo antes de ser destituido.

Precisamente a consecuencia del tal escándalo y de la situación precaria del ministro de agricultura, Valdez Angulo, durante esos meses se habían acumulado decenas de asuntos urgentes, entre ellos la exportación con fines científicos de unos 800 monos "pichico" (Saguinus). Estos estaban en Lima, esperando la firma de la resolución suprema que permitiría su traslado a un laboratorio en los EE UU, donde servirían para concluir los estudios para lanzar la primera vacuna contra la hepatitis. Esa operación patrocinada por la Organización Mundial de la Salud, estaba perfectamente encuadrada en las normas vigentes, es decir en un decreto supremo aprobado un año antes, por mi iniciativa, que prohibía la exportación comercial de fauna pero que, mediante resolución suprema, permitía excepciones de tipo científico como la que ocasionó todo el problema ${ }^{3}$.

Así es como, en el primer despacho que tuve con el nuevo ministro, llevé el asunto de los monos como prioridad absoluta. Me respondió que, coincidentemente, tendría su primer despacho semanal con el presidente al día siguiente y que abordaría el tema. Lo que sigue ya ha sido relatado (Dourojeanni, 1986, 1988) y resultó en que el 14 de noviembre de 1974 recibí una llamada furibunda del presidente que no me dejó responder nada coherente antes de colgar el teléfono y que, una hora más tarde, llegó a mi oficina el ministro Gallegos en persona, bastante descompuesto, diciéndome más o menos que ese había sido uno de los peores

\footnotetext{
${ }^{2}$ Fue una de las empresas estatales creadas en 1970 por el gobierno de Velasco Alvarado con la finalidad de aumentar la producción y productividad agraria, mejorar las condiciones en que se realiza la comercialización de los insumos y productos agropecuarios y asegurar el abastecimiento de los que resulten más necesarios y estabilizar su precio en el mercado interno. De una parte, EPSA no fue manejada adecuadamente y de otra fue afectada por la política de subsidios del gobierno (Chirinos y Chirinos, 1977). Una auditoría basada en criterios equívocos terminó con la Contraloría General de la República encontrando "pérdidas millonarias" en Epsa (se habló de 20\% del dinero circulante nacional) determinando la prisión preventiva de medio centenar de funcionarios de alto nivel del sector agrario y la destitución de sus funciones de algunos militares, incluido el ministro. El tiempo demostró que, en realidad ellos no eran responsables del problema. Por eso, finalmente, en el escándalo de Epsa no se acusó a ningún funcionario público, aunque si quedó en evidencia que hubo malos manejos en los molinos de arroz del norte, lo que no explicaba el monto "perdido". EPSA fue liquidada en 1979.

${ }^{3}$ Decreto Supremo 934-73 AG de 3 de octubre de 1973. La captura era permitida por resolución ministerial pero la exportación requería resolución suprema
} 
días de su vida, que el presidente lo trató de estúpido "por dejarse engañar" y que había recibido dos órdenes: la primera era, retornar los monos a la libertad. La segunda era destituirme o, si quería mantenerme en el puesto, eso sería bajo su propia responsabilidad.

Lo que yo no sabía, es que el presidente había sido informado, personalmente, por Felipe Benavides Barreda ${ }^{4}$ de que "esa exportación era un crimen ambiental”. Benavides, importador de armas británicas para la Marina de Guerra y aficionado a temas ambientales, participaba frecuentemente de juegos de carta con Velasco y, aprovechó una de esas reuniones para hacer esa falsa denuncia. Benavides supo del asunto porque tanto Carlos Ponce del Prado (Director de Conservación), como Antonio Brack (Subdirector de Fauna Silvestre), los responsables directos de la operación de los monos en la Dirección General Forestal y de Fauna que yo dirigía eran, asimismo, miembros de la organización no gubernamental Pro Defensa de la Naturaleza (Prodena) que era presidida por Benavides que, por entonces, declaraba ser amigo nuestro. No era la primera ni la última "traición" y difamación de Benavides a sus colegas de Prodena. Esos hechos fueron uno de tantos motivos por los que Brack y yo nos apartamos de Benavides. Ponce, diplomático por definición, resistió hasta la extinción de Prodena.

No se trata aquí de repetir la historia de la tan ridícula como épica "liberación" de los monos, lo que estuvo a cargo de Carlos Ponce. Pero la orden fue cumplida a pesar de que Gallegos entendió toda su irracionalidad y alto costo. En efecto, trasladar de vuelta a Iquitos los anima- les que habían estado enjaulados durante varios meses en Lima y liberarlos en la ribera del río Nanay, que es lo único que permitió hacer la Marina, que proporcionó la balsa, era condenarlos a la peor de las muertes. Pero, así fue.

Gallegos no obedeció la segunda orden. No me destituyó, pero mi personal y yo tuvimos que soportar toda clase de investigaciones e inspectorías a cargo de coroneles que no obedecían al ministro. Creo que éste entendía que sacarme del puesto dificultaría una larga agenda de acciones y proyectos que le habían llamado la atención, lo que incluía en primer lugar culminar los detalles para la aprobación de la nueva Ley Forestal y de Fauna Silvestre, el establecimiento de varias áreas naturales protegidas, el desarrollo del Programa Nacional de Manejo de la Vicuña, la operación del aserradero del Bosque Nacional de Iparía, negociaciones con numerosas cooperaciones técnicas internacionales, etc. Además, el tema forestal le interesó personalmente y, por eso, hubo varias ocasiones de interactuar con él, más aún porque él visitó, personalmente, muchas de las acciones forestales del Ministerio.

El proyecto de la nueva ley forestal y de fauna estaba pronto antes de la llegada del nuevo ministro y comenzó a pasar por los filtros habituales de la época, entre ellos el Comité de Asesores de la Presidencia (Coap). Hubo interminables discusiones con el ministerio de Pesquería que, bajo la gestión del general Javier Tantaleán Vanini y el enorme aporte económico de la pesca exhaustiva de la anchoveta, tuvo bastante influencia. Tantaleán no quería aceptar que se crearan áreas naturales protegidas marinas, como Paracas o en cuer-

\footnotetext{
${ }^{4}$ Benavides (1917-1991), una personalidad de la alta sociedad tradicional limeña, fue un defensor de la naturaleza destacando sus actuaciones para frenar la caza de ballenas y vicuñas y el establecimiento del Parque de las Leyendas (zoológico de Lima). Protagonizó varios debates públicos, entre ellos uno particularmente virulento con relación al aprovechamiento de la vicuña contra Antonio Brack Egg, quien después fue el primer ministro de ambiente del Perú.
} 
pos de agua, como Pacaya y Samiria o el Titicaca, y tampoco aceptaba que tortugas y lagartos negros o blancos, entre otras especies, dependieran del sector Agrario. Finalmente, se logró el acuerdo que existe hasta hoy: Dependen del subsector forestal las especies cuya reproducción y postura se realiza en tierra firme. Había muchísimas otras discusiones relacionadas a la cuestión del uso de la tierra a resolver con la Reforma Agraria y otros, de tipo ideológico, con el Sistema Nacional de Apoyo a la Movilización Social (Sinamos), el brazo político de los militares revolucionarios. Varios de los temas discutidos repercutieron en la prensa, donde recibía bastante crítica de la por entonces muy activa prensa izquierdista que, sólo en teoría, apoyaba al gobierno.

Transcurrieron algunos meses difíciles hasta que un día Gallegos me dijo, más o menos: "doctor, vamos a enfrentar el toro por las astas. El presidente sigue teniéndolo entre ceja y ceja y eso no nos deja trabajar. Él jamás deja de ver un programa político semanal, en el Canal Panamericana Televisión, que se llama "El Público Quiere Saber" (figura 2) . Quiero que Ud. participe presentando la nueva ley. Estoy seguro de que si el presidente lo escucha va a aprobarlo. Puede llevar a otro funcionario si así lo desea". El formato del programa era un círculo con el participante interrogado al medio y de 10 a 20 personalidades planteando preguntas durante bastante más que una hora continua. Decidí llevar a Antonio Brack, que ya era un gran orador, para tratar de los temas de fauna y yo me encargué de los temas forestales y políticos. Pues, así fue y Brack y yo hicimos lo que pudimos. Esa misma noche Gallegos me llamó y dijo algo así como: "Doctor, todo resuelto. El presidente gustó mucho de la presentación y defensa que hicieron".

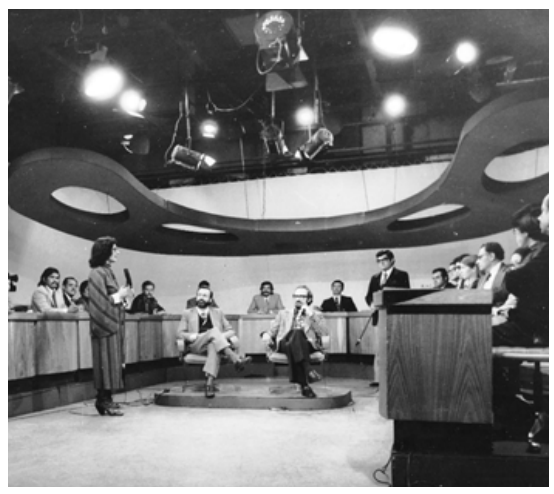

Figura 2. "El Público quiere Saber": El programa político de Panamericana Televisión que, en 1974, permitió avanzar con la Ley Forestal y de Fauna. Antonio Brack y Marc Dourojeanni fueron los defensores del proyecto.

Poco después, el 13 de mayo de 1975 la Ley Forestal y de Fauna Silvestre fue aprobada. El capítulo de áreas protegidas de esa ley y su reglamento se convirtieron, sin cambios sustanciales, en la ley vigente de áreas naturales protegidas aprobada en 1997.

De allí en adelante todo el trabajo de la Dirección General Forestal y de Fauna fue facilitado y acelerado pues, finalmente, el presidente pasó a aprobar, sin crear obstáculos, los dispositivos legales que sustentaban las propuestas del subsector forestal. El ministro permitió, por primera vez, que los directores de las cuatro regiones agrarias de la Selva sean de los cuadros forestales rompiendo la tradición de que sean de las direcciones generales de agricultura o ganadería. Consiguió hacer aprobar el establecimiento de los parques nacionales Huascarán (01/07/1975) y Cerros de Amotape (22/07/1975), de la Reserva Nacional Paracas (25/09/1975) y del Coto de Caza El Angolo (01/07/1975), este último luego de complejas negociaciones con los Romero, dueños de las haciendas expropiadas, y con la Dirección General de Reforma Agraria. En ese lapso se prepararon varios otros proyectos de áreas protegidas (Lachay, Titicaca, Salinas y Aguada Blanca, que fueron aprobadas por el 
ministro general Luis Arbulú Ibáñez, el sucesor de Gallegos, quien también tuvo una actitud muy positiva hacía el subsector forestal.

Cupo a Gallegos, asimismo, dar un gran impulso oficial a la conservación de la vicuña, creando el Programa Nacional de Manejo de la Vicuña, poniendo a su frente a Antonio Brack, con fuerte apoyo de la cooperación del gobierno alemán. Felipe Benavides ya había comenzado su campaña contra Antonio Brack con relación al manejo de la vicuña, pero no se atrevió a hacerlo públicamente mientras Gallegos fue ministro. Inició sus ataques más virulentos (Dourojeanni, 2018) pocos meses después de que Arbulú asumió el cargo. La saga del problema de los monos dio lugar a otra acción muy importante, el Programa de Primates no Humanos, con apoyo de la Organización Panamericana de la Salud y del Instituto Veterinario Tropical y de Altura. En todas esas iniciativas el ministro participó activamente y brindó todo el apoyo que pudo conseguir tanto en su sector como en otros ministerios.

Otra iniciativa excepcional de Gallegos fue combinar, con el general Jorge Chávez Quelopana, por entonces director del Centro de Altos Estudios Militares (Caem), el ofrecimiento de un curso de ecología y defensa nacional que durante varios años estuvo a mi cargo, pasando luego a ser ofrecido por varios otros especialistas. Dicho sea de paso, Chávez Quelopana, cuando jefe del Instituto Nacional de Planificación, fue extremamente receptivo a los temas ambientales y a las ideas para el desarrollo más sensato de la Amazonia. El curso comenzó con dos horas de clase, pero antes de fines de la década de 1970 ya era un curso obligatorio con 20 horas de clase lo que contribuyó, sin duda, a concientizar a los futuros jefes militares y altos funcionarios civiles a preocuparse más por la temática ambiental.

Finalmente, es en gran medida al general Gallegos a quien se debe la creación de la Policía Forestal de la Guardia civil que ha dado lugar a la actual Policía Ecológica del Perú. Fue de él la idea de pedir el destaque de un oficial superior de la Guardia Civil a mi despacho para facilitar las coordinaciones de apoyo de ese cuerpo para la defensa de las áreas protegidas y de la fauna silvestre, especialmente de la vicuña. A poco de llegar, el por entonces mayor Jesús, Sánchez Bocanegra pronto realizó que la mejor solución sería crear un cuerpo policial forestal especializado dentro de la Guardia Civil. Expusimos la idea al ministro, quien de inmediato entró en contacto con el Director General de la misma, el general Gastón Zapata de la Flor y, después de un par de reuniones se comenzó el trabajo con apoyo adicional del coronel Pedro Reynoso, destacado por la Guardia Civil para ese fin. Fue muy difícil y requirió un esfuerzo muy grande, pues, a pesar del respaldo de los jefes, la idea requería mucho planeamiento y la solución previa de diversos problemas legales y, también académicos. Además, el Ministerio de Agricultura debería transferir fondos a la Guardia Civil para la operación, algo que nunca es fácil. Pero, el cuerpo de Policía Forestal fue formalmente creado el 16 de febrero de 1976 y la primera promoción egresó en 1977.

Lo arriba escrito no revela toda la dimensión del aporte de Gallegos a la conservación del patrimonio natural del Perú, realizado en menos de 20 meses de trabajo en el que prestó atención a inúmeros otros problemas ambientales y del sector agrario, especialmente a la reforma agraria que aún estaba muy activa. Tampoco significa que otros ministros militares no apoyaran el tema. Al contrario, tanto los generales Valdez Angulo como, es- 
pecialmente, Arbulú Ibañez hicieron mucho por el tema. Y tampoco significa que los ministros militares hicieron más o fueron mejores que muchos ministros civiles. En efecto, ministros de agricultura civiles como Carlos Amat y León, Álvaro Quijandría Salmón y Nils Ericsson Correa, entre otros, siempre favorecieron la conservación del patrimonio natural. Lo único que se ha pretendido es destacar la figura del general Enrique Gallegos Venero que, en entorno del ambientalismo peruano, es injustamente desconocido.

\section{Gallegos no fue el único}

Son varios los hechos importantes para la conservación del patrimonio natural peruano que fueron realizados durante gobiernos militares. Puede, por cierto, alegarse justamente que en esos periodos no democráticos es más fácil tomar medidas que, de lo contrario, serían motivo de interminables discusiones y deformaciones. Eso es verdad. Pero lo que no puede negarse es que hubo militares interesados en hacerlas. Y el primero fue nada menos que el dictador general Simón Bolívar, quien en 1825 emitió la primera medida del nuevo Perú para la protección de la vicuña, prohibiendo su matanza y otorgando compensaciones a los que las cuidan.

En esta nota no se ha hecho un recuento de todas las medidas ambientales adoptadas durante regímenes militares a lo largo de la historia republicana. Apenas se mencionan algunas de las más importantes y recientes, además de tratar sólo de las referidas al patrimonio natural, como las dos primeras leyes forestales del Perú, la de 1963 y la de 1975 que fueron hechas durante y aprobadas por regímenes militares. La de 1963, aprobada bajo el gobierno de la junta militar del general Lindley, recibió apoyo del entonces ministro de agricultura, el general Alfonso Terán Brambilla. También fue en 1963 que se creó el primer Servicio Forestal y de Caza. Y, muy importante es recordar que fue ese mismo año y régimen que se crearon 13 bosques nacionales, abarcando unos seis millones de hectáreas, muchos de los cuales, como el del Manu, fueron la base de los parques y reservas nacionales hoy existentes.

Un hecho curioso es que el primer bosque nacional creado en el Perú, en 1949, el de Tumbes, fue iniciativa de otro militar, el general Carlos Miñano Mendocilla, bajo el gobierno de Manuel A. Odría. Es importante recordar que eso ocurrió más de una década antes de que se creara el primer parque nacional y antes de que se establecieran más bosques nacionales. El gobierno de Odría también decretó diversas medidas en favor de la fauna silvestre.

Asimismo, se debe recordar que el Parque Nacional del Manu, al que el presidente Belaúnde se opuso tenazmente (Dourojeanni, 2017), fue creado por el gobierno de Velasco Alvarado cuando el ministro de agricultura era el general Enrique Valdez Angulo. Otras cinco áreas protegidas (Lachay, Titicaca, Calipuy, Salinas y Aguada Blanca y Sunchubamba) también fueron creadas durante el régimen militar por los ministros Arbulú y Pérez Egaña, después de la salida de Gallegos. Y todas las diez áreas naturales protegidas que fueron establecidas por Fernando Belaúnde y Alan García en la década de 1980 eran proyectos elaborados durante el régimen militar. También debe llevarse en cuenta que la Ley de Comunidades Nativas y Desarrollo Agropecuario de la Selva (figura 3), de 1974, ha dado lugar a la conservación en la actualidad de más de 12 millones de hectáreas en forma de comunidades nativas (Dourojeanni, 2009; 2018).

Y estos hechos no son peculiarmente peruanos. Una breve revista de lo ocurrido en el Brasil, por ejemplo, revela lo mismo. En efecto, la 
primera secretaria nacional del ambiente, hoy ministerio del ambiente de ese país, fue creada en el momento más duro del régimen militar de Ernesto Geisel (1974-1979) y continuó bajo el mandato del general Joao Figueiredo (19791985). Durante estos dos gobiernos se establecieron más de 15 millones de hectáreas como parques, reservas y estaciones ecológicas, principalmente en la Amazonía y el Cerrado, además de diversos proyectos importantes de manejo de fauna como el de las tortugas marinas y de río, manatíes y de anillado de aves, que continúan vigente (Pádua y Coimbra,1979; Urban,1998; Pádua, 2015) Fueron numerosas las leyes de ese periodo en favor del ambiente, incluida la creación del poderoso Consejo Nacional del Ambiente, que permitía una amplia intervención de la sociedad civil y que moderaba el poder del propio ministro.

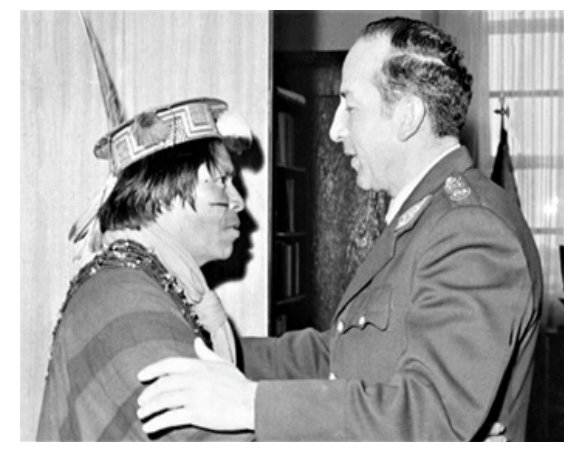

Figura 3. Cupo a Gallegos comenzar a implementar la nueva Ley de Comunidades Nativas de 1974, que permitía la titulación de comunidades nativas. Y lo hizo con legítimo entusiasmo y buenos resultados.

Dos de los más famosos y apreciados ambientalistas brasileños fueron, ambos, vicealmirantes. En efecto, José Luiz Belard e Ibsen Gusmão Câmara no tan solo propusieron y consiguieron el establecimiento de áreas de conservación marina y, en el caso del segundo, uno de los mayores y más exitosos programas de conservación de ballenas, sino que ambos fueron mentores de varias generaciones de ambientalistas brasileños (Urban, 1998). Gusmão Câmara, en especial, facilitó también la creación de áreas naturales protegi- dos en otros biomas, como en la Amazonia, el Cerrado, el Pantanal y la Mata Atlántica e, inclusive durante gobiernos militares, se opuso tenazmente a obras que, como un proyecto de nuevo aeropuerto en Sao Paulo, afectaría una importante área de bosque nativo. Por todo eso fue conocido como "almirante verde" (Dourojeanni, 2006, Ortiz, 2013). Retirados, ambos continuaron luchando por mejorar el ambiente en el Brasil hasta sus últimas horas.

Lo que he observado a lo largo de mis contactos con militares en relación a temas ambientales es que ellos, definitivamente más que los civiles, conocen el territorio de sus países. En el Perú todos ellos han servido en Costa, Sierra y Selva, viviendo largos periodos en cada región y, cuándo se les habla de un sitio u otro a preservar, ellos usualmente conocen el lugar o saben de él. Además, hablando de áreas naturales protegidas, ellos tienen por profesión, un buen sentido de lo que es, en realidad, un territorio, así como de la defensa de esos espacios. De otra parte, ellos no pretenden ser otra cosa que lo que son y, por eso, escuchan y procuran entender los argumentos, no teniendo rechazos apriorísticos, lo que es común en ministros agrónomos, abogados o economistas, que casi siempre pretenden saber mucho de todo. Y, con eso, no implico que es mejor tener ministros militares. Apenas se explica por qué ellos, cuando lo fueron, apoyaron mucho la cuestión de defensa del patrimonio natural.

Para terminar, como ya dicho, el único propósito de esta nota es recordar que la preocupación por un ambiente mejor no es exclusividad de las personas que no usan uniforme. Muchas obras de tipo ambiental importante también ocurrieron bajo gobiernos militares y muchos militares en el Perú, como en otros países, fueron actores claves de estos hechos, bien sea con iniciativas propias o, más 
frecuentemente, escuchando, entendiendo y adoptando las propuestas de los profesionales especializados, a las que permitieron y ayudaron a concluir exitosamente sus propuestas.

También, con esta nota, me propuse rendir un justo homenaje al general Enrique Gallegos Venero quien en muy poco tiempo hizo más que lo que el mero deber le exigía con relación a la naturaleza en el Perú.

\section{REFERENCIAS BIBLIOGRÁFICAS}

BID. (2002). Diálogos de Política Social e Ambiental: Aprendendo com os Conselhos Ambientais Brasileiros. Primeira Parte. Banco Interamericano de Desenvolvimento. Brasília. 278p.

Chirinos, Lizares y G y Chirinos Soto E. (1977). El Septenato 1968-1975. Edit. Alfa. Lima.

Dourojeanni, M. J. (1986). El general, los monos y Felipillo. Editorial en el Diario La República. Lima. 30 de mayo de 1986.

Dourojeanni, M. J. (1988). Si el Árbol de la Quina Hablara. Lima, Fundación Peruana para la Conservación de la Naturaleza, ProNaturaleza. 312 p. https://www.academia. edu/42145692/Si_el_\%C3\%A1rbol_de_la_ quina_hablara.

Dourojeanni, M. J. (2006). O almirante verde. Oeco, Rio de Janeiro. https://www.oeco.org.br/ colunas/marc-dourojeanni/16393-oeco-17189/

Dourojeanni, M. J. (2009). Crónica Forestal del Perú. Universidad Nacional Agraria La Molina, Editorial San Marcos, Lima. 727 p. https://www.academia.edu/14408042/Cr\%C3\%B3nica_Forestal_del_Per\%C3\%BA
Dourojeani, M J. (2017). Belaúnde en la Amazonia. CAAP. Lima. https://www.caaap. org.pe/2017/06/12/belaunde-en-la-amazonia-por-marc-j-dourojeanni/

Dourojeanni, M J. (2018). Áreas Naturales Protegidas del Perú: El Comienzo. Fondo Editorial Univ. Nac. Educ. Guzmán y Valle y Grijley, Lima. 330 p. https://www.academia. edu/37923859/\%C3\%81REAS_NATURALES_PROTEGIDAS_DEL_PER\%C3\%9A_ EL_COMIENZO

Dourojeanni,M. J. y C Ponce. (1978). Los Parques Nacionales del Perú. Madrid, España. Instituto de la Caza Fotográfica (INCAFO). Colección "La Naturaleza en Iberoamerica” 224 p. 317p. https://www.academia. edu/43865362/Parques_Nacionales_del_ Per $\%$ C3\%BA

Ortiz, F. (2013). Almirante Ibsen: uma vida dedicada ao meio ambiente. OEco, Rio de Janeiro. https://www.oeco.org.br/reportagens/27829-almirante-ibsen-uma-vida-dedicada-ao-meio-ambiente/

Pádua, M. T. (2015). Conservando a Natureza d Brasil. Fundação Grupo o Boticario de Conservação a Natureza. Curitiba. 215 p.

Pádua, M. T. y Coimbra, A. (1979). Os Parques Nacionais do Brasil. Madrid, España. Instituto de la Caza Fotográfica (INCAFO). Colección "La Naturaleza en Iberoamerica" 223p.

Toche, E. (2008). Guerra y democracia: los militares peruanos y la construcción nacional. DescolClacso, Lima. 308 p. http://biblioteca.clacso.edu.ar/clacso/becas/20120419125101/medrano.pdf Urban, T. (1998). Saudade do Matâo. 1998. E.D. UFPR. Curitiba. 371p. 